\title{
Production of avirulent mutants of Mycobacterium bovis with vaccine properties by the use of illegitimate recombination and screening of stationary-phase cultures
}

AgResearch, Wallaceville Animal Research Centre, PO Box 40063, Upper Hutt, New Zealand

\author{
D. M. Collins, T. Wilson, S. Campbell, B. M. Buddle, B. J. Wards, G. Hotter \\ and G. W. de Lisle
}

Author for correspondence: D. M. Collins. Tel: +64 4922 1310. Fax: +64 49221380. e-mail: desmond.collins@agresearch.co.nz

\begin{abstract}
A better tuberculosis vaccine is urgently required to control the continuing epidemic. Molecular techniques are now available to produce a better live vaccine than BCG by producing avirulent strains of the Mycobacterium tuberculosis complex with known gene deletions. In this study, 1000 illegitimate recombinants of Mycobacterium bovis were produced by illegitimate recombination with fragments of mycobacterial DNA containing a kanamycin resistance gene. Eight recombinant strains were selected on the basis of their inability to grow when stationary-phase cultures were inoculated into minimal medium. Five of these recombinants were found to be avirulent when inoculated into guinea pigs. Two of the avirulent recombinants produced vaccine efficacy comparable to BCG against an aerosol challenge in guinea pigs with $M$. bovis. One of these recombinants had an inactivated gInA2 gene encoding a putative glutamine synthetase. Transcriptional analysis showed that inactivation of gInA2 did not affect expression of the downstream gInE gene. The other recombinant had a block of 12 genes deleted, including the sigma factor gene sigG. Two avirulent recombinants with an inactivated pckA gene, encoding phosphoenolpyruvate carboxykinase which catalyses the first step of gluconeogenesis, induced poor protection against tuberculosis. It is clear that live avirulent strains of the $M$. tuberculosis complex vary widely in their ability as vaccines to protect against tuberculosis. Improved models may be required to more clearly determine the difference in protective effect between BCG and potential new tuberculosis vaccines.
\end{abstract}

Keywords: tuberculosis, Mycobacterium tuberculosis, vaccines, virulence, BCG, guinea pig

\section{INTRODUCTION}

Tuberculosis exacts enormous suffering and death on a global basis. Most disease is caused by Mycobacterium tuberculosis, but in many parts of the world a significant amount of disease is caused by the very closely related organisms Mycobacterium africanum and the major animal pathogen Mycobacterium bovis. Together with a few less important species, these organisms are collectively referred to as the M. tuberculosis complex.

Abbreviation: PPD, purified protein derivative.
Many factors contribute to this global burden of tuberculosis, but one cause is undoubtedly the lack of a more effective vaccine. The current vaccine, Bacille Calmette-Guérin (BCG), is an attenuated strain of Mycobacterium bovis that was developed 80 years ago by the simple technique of subjecting a virulent $M$. bovis strain to serial culture in vitro for 13 years (Brewer \& Colditz, 1995). BCG has become accepted to the extent that there have been at least 3 billion doses administered, more than any other vaccine (Bloom \& Fine, 1994). While BCG undoubtedly prevents childhood forms of the disease, it provides only limited protection against the vast majority of disease and in the most recent large 
trial it provided no protection at all (Bloom \& Fine, 1994; Brewer \& Colditz, 1995). Although it has been difficult to demonstrate that BCG has had any major effect on reducing the incidence of disease (Fine, 1995), the very wide and continuing use of BCG and its ability to limit the severity of disease in animal models has made it the gold standard against which new experimental vaccines are compared.

In the last 10 years, an increased concern about tuberculosis together with many developments in immunology and molecular biology has aroused intense interest in producing better vaccines against the disease. There is general agreement that a successful vaccine will need to induce a type 1 immune response, but the detailed combination of responses that are required to induce optimum levels of protective immunity is not known (Snewin et al., 2000). This has made it difficult to specify which features of a vaccine are most important and therefore which particular type of vaccine is likely to prove most effective. A large range of possible types of both living and non-living vaccines are being actively researched (Collins, 2000; Snewin et al., 2000) and different types of vaccines may be useful for different purposes.

One approach is to modify the current vaccine and already a genetically modified form of BCG has been reported to produce better protection than BCG (Horwitz et al., 2000). The genetic causes of attenuation in BCG remained completely unknown until 6 years ago when it was shown that a block of nine genes, including the esat6 gene, was missing in BCG but was present in other strains of the M. tuberculosis complex (Mahairas et al., 1996). More recently, specific inactivation of the esat6 locus was shown to cause partial, but not complete attenuation of an M. bovis strain (Wards et al., 2000), and other blocks of genes that are absent (Behr et al., 1999; Gordon et al., 1999) or duplicated (Brosch et al., 2002) in BCG have been identified. Add to this the likely presence of mutated genes which are yet to be identified and it is evident that considerable further work will be required to elucidate the role of the many genetic differences between BCG and clinical tuberculosis strains.

An alternative method of making a new live tuberculosis vaccine is to attenuate a virulent strain of the $M$. tuberculosis complex by introducing one or more easily identifiable mutations and several groups have shown that vaccine candidates can be produced in this way (de Lisle et al., 1999; Jackson et al., 1999; Hondalus et al., 2000). In the early stages of carrying out allelic exchange of ahpC in an M. bovis strain (Wilson et al., 1997), using an approach that was eventually successful (Collins, 2001), we had produced moderate numbers of illegitimate recombinants and investigated their potential use as tuberculosis vaccines. A total of 440 illegitimate recombinants were screened on minimal medium and four mutants were selected, all of which were found to be attenuated in guinea pigs. When guinea pigs inoculated with these mutants were challenged with virulent
M. bovis, two of the mutants induced a level of protection that was in some respects better than that induced by BCG, although the differences were not significant (de Lisle et al., 1999). In one of these mutants, a putative undecaprenol kinase gene was interrupted by illegitimate insertion of a DNA fragment that accompanied a $2 \mathrm{bp}$ chromosomal deletion and, in the other, DNA fragment insertion was accompanied by a large DNA deletion of $15 \mathrm{~kb}$ containing 12 genes. None of the four mutants appeared to have any interrupted genes encoding enzymes for common metabolic pathways that are often associated with auxotrophy, such as amino acid, purine, pyrimidine or co-factor synthesis. Subsequently, we found these mutants were not true auxotrophs as 2-week-old cultures in complete medium were able to grow when inoculated into minimal medium. Re-examination of procedures revealed the mutants in the study had been subcultured into minimal medium from 5- to 10-week-old cultures in complete medium, at a stage when they would have been in stationary growth. Since the ability of strains of the M. tuberculosis complex to survive in the host in a stationary or lowered metabolic state is regarded as a crucial facet of tuberculosis pathogenesis (Colston \& Cox, 1999), we reasoned that it might be desirable to have a vaccine strain without this ability.

Regardless of this explanation, the use of 5- to 10-weekold cultures had produced two mutants with vaccine effectiveness at least equal to that of BCG, so a similar approach was used to produce mutants in this study. Eight recombinants were obtained by screening 1000 illegitimate mutants of $M$. bovis and these were produced by using either the original $a h p C$ fragment or a second fragment made from another $M$. bovis gene. Identification of the mutated regions and virulence and vaccine testing of the strains was performed and two strains with vaccine properties at least as good as those of BCG were obtained.

\section{METHODS}

M. bovis strains and culture conditions. M. bovis ATCC 35723, the strain used for illegitimate recombination, and $M$. bovis WAg201, the strain used to challenge animals in vaccine studies, were cultured in Middlebrook 7H9 (Difco) liquid medium supplemented with albumin/glucose complex (ADC; Difco), $0.05 \%$ Tween 80 and $0.4 \%$ sodium pyruvate, and on Middlebrook 7H11 (Difco) solid medium supplemented with ADC and $0.4 \%$ sodium pyruvate. Recombinants were isolated on 7H11 medium supplemented with $2.5 \%$ bovine serum and $20 \mu \mathrm{g}$ kanamycin $\mathrm{ml}^{-1}$. For screening of recombinants in minimal medium, $5 \mu \mathrm{l}$ of a 5- to 8-week-old culture in supplemented Middlebrook $7 \mathrm{H} 9$ medium was inoculated into both supplemented Middlebrook 7H9 medium and into Proskauer \& Beck minimal liquid medium (Darzins, 1958) supplemented with $0.05 \%$ Tween 80 and $0.2 \%$ sodium pyruvate. Recombinants that did not grow in minimal medium were rescreened twice to confirm their stability. For culture of $M$. bovis from guinea pig spleens, half the spleen was homogenized with $20 \mathrm{ml}$ water for $1 \mathrm{~min}$. This was filtered through sterile cheese cloth and centrifuged at $3500 \mathrm{~g}$ for $20 \mathrm{~min}$. The pellet was resuspended in $0.5 \mathrm{ml}$ water and 


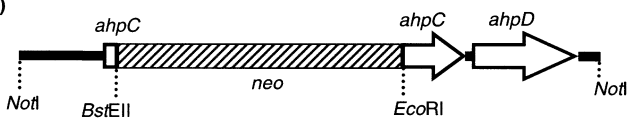

$\overline{250 \mathrm{bp}}$

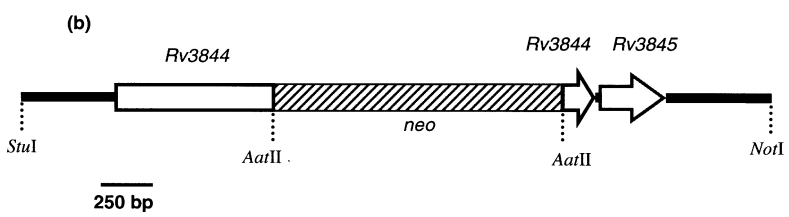

Fig. 1. Constructs used for illegitimate recombination. (a) DNA fragment containing ahpClahpD and neo (kanamycin resistance); (b) DNA fragment containing Rv3844/Rv3845 genes and neo.

aliquots were plated onto Middlebrook $7 \mathrm{H} 11$ supplemented with $0.6 \mathrm{ml}$ oleic acid $\mathrm{l}^{-1}, 50 \mathrm{~g}$ bovine serum albumin $\mathrm{l}^{-1}, 20 \mathrm{~g}$ glucose $\mathrm{l}^{-1}, 7 \cdot 7 \mathrm{~g} \mathrm{NaCl}^{-1}, 0 \cdot 4 \%$ sodium pyruvate, $0 \cdot 5 \%$ lysed sheep red blood cells, $10 \%$ bovine serum, $10 \mu \mathrm{g}$ fungizone $\mathrm{ml}^{-1}, 200 \mathrm{IU}$ polymixin B sulphate, $100 \mu \mathrm{g}$ tarcarcillin $\mathrm{ml}^{-1}$ and $10 \mu \mathrm{g}$ trimethoprim $\mathrm{ml}^{-1}$.

Techniques for making recombinants and determining mutation sites. The DNA fragment containing the interrupted $a h p C$ gene (Fig. 1a) used for illegitimate recombination was prepared by NotI digestion as described previously (Wilson et al., 1997). A DNA fragment containing the Rv3844 gene was also prepared. Briefly, a $2593 \mathrm{bp}$ region of M. bovis (homologous to $M$. tuberculosis at position 13157-15749 of Mtcy1A6; GenBank accession no. Z83864) was amplified using PCR primers containing PacI sites and cloned into pUHA9, a derivative of pBluescript KSII + (Stratagene) which contains a PacI cloning site. A kanamycin resistance gene (neo) was inserted at the Aat II site of $R v 3844$ by appropriate DNA digestion, blunt-end ligation and cloning. For electroporation, the plasmid containing this construct was digested with $S t u \mathrm{I}$ and NotI which cut at positions equivalent to 13257 and 15209 respectively in the 13157-15749 fragment of Mtcy1A6 to produce a $3.4 \mathrm{~kb}$ fragment (Fig. 1b). The $a h p C$ and $R v 3844$ plasmid digests were precipitated and washed in ethanol to remove electrolytes and electroporated into $M$. bovis using a high efficiency technique at $37^{\circ} \mathrm{C}$ as described previously (Wards \& Collins, 1996). The electroporated cells were cultured on solid medium containing kanamycin and individual colonies were subcultured in liquid medium containing $20 \mu \mathrm{g}$ kanamycin $\mathrm{ml}^{-1}$.

DNA was extracted (van Soolingen et al., 1991) from liquid cultures of strains identified by their failure to grow on minimal medium, digested with EcoRI, separated on a $20 \mathrm{~cm}$ $1 \%$ agarose gel and transferred to nylon (Zeta probe; BioRad) by Southern blotting. Nylon filters were hybridized with probes made by incorporating $\left[{ }^{32} \mathrm{P}\right] \mathrm{dCTP}$ into a plasmid containing the neo gene by nick translation (Life Technologies). To determine the site of insertion of the DNA fragment in the chromosome of a recombinant, chromosomal DNA was digested with a restriction enzyme that did not cut within the inserted fragment (XhoI for the Rv3844 fragment and $E c o$ RI or BamHI for the $A b p C$ fragment), ligated into the appropriate site of pBluescript $\mathrm{KSII}+$, electroporated into Escherichia coli XL-1 Blue MR and plated on Luria-Bertani medium containing $25 \mu \mathrm{g}$ kanamycin $\mathrm{ml}^{-1}$ and $50 \mu \mathrm{g}$ ampicillin $\mathrm{ml}^{-1}$. The junction regions of the fragment and chromosome were sequenced, using appropriate primers directed outwards from the vector sequence into the mycobacterial sequence. DNA sequences were analysed using the programs of the Genetics Computer Group (Madison, WI, USA) and compared to GenBank (www.ncbi.nlm.nih.gov) and Sanger (www.sanger.ac.uk) databases.

Transcription of $\boldsymbol{g} \ln \mathbf{A 2}$. Exponential-phase cultures of $M$. bovis ATCC 35723 and WAg530 were harvested by centrifugation and RNA was extracted by cell disruption of $10^{8}$ c.f.u. in the presence of $1 \mathrm{ml}$ Trizol (Invitrogen) and $0.5 \mathrm{ml}$ zirconium beads. After Trizol extraction, residual DNA was removed by incubation in DNaseI (Invitrogen) and RNA was further purified using an RNeasy Mini Kit (Qiagen). Reverse transcription was performed using gene-specific primers (designated RT-PCR in Table 1) and C. therm polymerase (Roche), according to the manufacturer's instructions. PCR was performed in either a GeneAmp PCR System 9600 (Applied Biosystems) or a GeneAmp PCR System 9700 (Applied Biosystems) in 9600 emulation mode using the following programme: 1 cycle of $94^{\circ} \mathrm{C}$ for $5 \mathrm{~min} ; 32$ cycles of $94^{\circ} \mathrm{C}$ for $45 \mathrm{~s}, 60^{\circ} \mathrm{C}$ for $30 \mathrm{~s}, 72^{\circ} \mathrm{C}$ for $60 \mathrm{~s}$; and 1 cycle of $72{ }^{\circ} \mathrm{C}$ for $7 \mathrm{~min}$.

Determination of virulence. All animal work was approved by the institution's Animal Ethics Committee. The virulence of each M. bovis strain was tested in three female DuncanHartley guinea pigs, as described previously (Wilson et al.,

Table 1. Primers used for RT-PCR of the glnA2 locus

\begin{tabular}{|llll|}
\hline Gene & Primer & \multicolumn{1}{c|}{ Use } & \multicolumn{1}{c|}{ Nucleotide sequence } \\
\hline$g \ln E$ & DMC419 & RT-PCR* & GGGCATACGACATCCAGTCC \\
$g \ln E$ & DMC420 & PCR & TGGGCGAAGGCGGCTACATC \\
$g \ln A 2-g \ln E$ & DMC421 & RT-PCR & CTCGTCCCATCCGGTGTCTG \\
$g \ln A 2-g \ln E$ & DMC422 & PCR & AGGCCGAGGACAACGTATGG \\
$g \ln A 2$ post-mutation & DMC527 & RT-PCR & GGTAGTTCGCCCACTCCGTG \\
$g \ln A 2$ post-mutation & DMC451 & PCR & TACACGCCGCACAAGACCTC \\
$g \ln A 2$ pre-mutation & DMC452 & RT-PCR & ACCCACTTCCGACAGCTGCA \\
$g \ln A 2$ pre-mutation & DMC453 & PCR & GCTACGTGCATCCCGAAATC \\
\hline
\end{tabular}

*Primer used for reverse transcription as well as PCR. 
1995). Animals were inoculated by injecting $10^{6}$ c.f.u. subcutaneously into their flank and were sacrificed at 8 weeks. The assessment of virulence was based on the presence of visible lesions of tuberculosis, particularly in the spleen. All guinea pigs were skin-tested by the intradermal injection of 4 units $(0.1 \mathrm{ml})$ of bovine purified protein derivative (PPD; Agriquality) immediately prior to inoculation with M. bovis and immediately prior to the animals being euthanased.

Determination of vaccine efficacy. For vaccination studies, groups of six Duncan-Hartley guinea pigs were vaccinated by subcutaneous injection in the right flank with $10^{5}$ c.f.u. of one of the avirulent recombinant strains. A group of 12 animals was vaccinated with BCG and a control group of 12 animals was not vaccinated. Eight weeks post-vaccination, all animals were challenged with a single cell suspension of $M$. bovis W Ag201 prepared by sonication for $30 \mathrm{~s}$ and filtration through an $8 \mu \mathrm{m}$ membrane filter. Guinea pigs were infected via the respiratory route by using an aerosol chamber which produces droplet nuclei of the size appropriate for entry into alveolar spaces (McMurray et al., 1985; Wiegeshaus et al., 1970). The concentration of viable $M$. bovis in the nebulizer fluid was empirically adjusted to result in the inhalation and retention of $2-10$ viable organisms per guinea pig. The aerosolized solution contained approximately $4 \cdot 8 \times 10^{4}$ c.f.u. $\mathrm{ml}^{-1}$. The requirement for this challenge dose had previously been estimated from the number of primary tubercles observed grossly in the lungs of non-vaccinated guinea pigs at 4 weeks post-infection. Five weeks after challenge, the animals were euthanased and autopsied, and body weight and gross pathology were recorded. Samples of spleen were subjected to mycobacterial culture and enumeration. Delayed type hypersensitivity to tuberculin injected intradermally was measured immediately before vaccination and prior to the animals being sacrificed using bovine PPD. Statistical analyses by ANOVA were performed on spleen weights and on $\log _{10}$ transformations of spleen and lung bacterial counts and numbers of macroscopic lesions in the spleen.

\section{RESULTS AND DISCUSSION}

\section{Isolation of recombinants and determination of mutation sites}

Five electroporations into M. bovis were performed for each fragment and a total of 1000 recombinants were recovered, 325 from the $a h p C$ fragment electroporations and 675 from the $R v 3844$ fragment electroporations. Strains that were unable to grow in minimal medium, but did grow in the supplemented Middlebrook 7H9 medium were rescreened twice and eight recombinants were obtained. A Southern blot hybridization of DNA from these eight strains and one recombinant that failed the selection is shown in Fig. 2. Subsequent testing of actively growing cultures of these eight strains showed that none of them was an auxotroph, although WAg530 and WAg531 did grow more slowly in minimal medium than the other recombinants. While none of the recombinants had identical insertions, analysis showed that in some cases insertion into the same region of the chromosome had occurred.

The fragment insertion site in the M. bovis chromosome of each of the eight recombinants was determined after cloning a region of the chromosome which contained

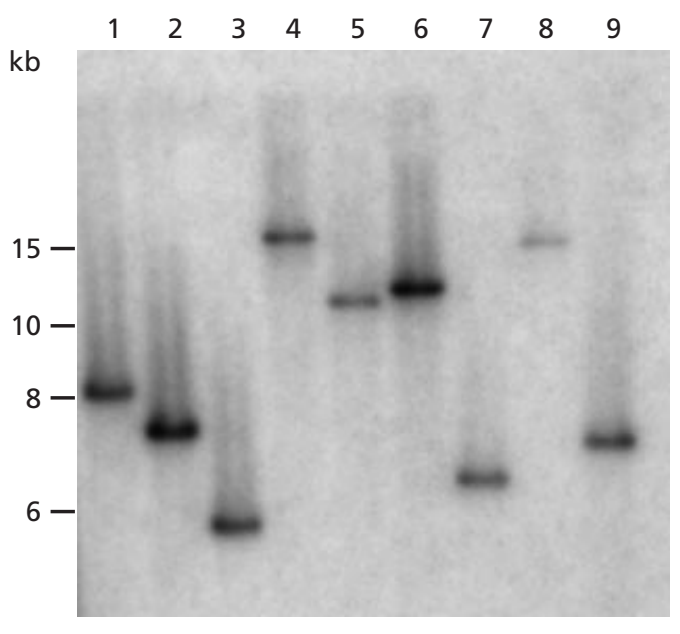

Fig. 2. Southern blot hybridization after EcoRI digestion and probing with neo of eight recombinants that were unable to grow in minimal medium (lanes 1-5, 7-9) and one recombinant that failed the screen and grew in minimal medium (lane 6).

the inserted fragment and flanking sequences. The initial sequencing primers used were located near each end of the fragment with their $3^{\prime}$ ends directed outwards so that the DNA which flanked the inserted fragment would be sequenced. In some of the recombinants there had been a deletion of the inserted fragment such that one of the initial primers did not bind. Other primers were subsequently used that were complementary to sequences further into the fragment until DNA sequence flanking both sides of the fragment was obtained. The insertion sites of the eight recombinants are given in Table 2 relative to the $M$. tuberculosis genome sequence in the Sanger database. The size of the DNA that was deleted from the chromosome during the insertion and the mean number of spleen lesions per guinea pig are also given. The chromosomal regions in which the fragments inserted and an illustration of the genes affected are shown in Fig. 3. The disruption ranged from one gene in WAg527 to 12 genes in WAg526. Three recombinants (WAg527, WAg528, WAg531) had insertions in the same region of the chromosome with deletions ranging from zero to almost $11 \mathrm{~kb}$ (Fig. 3a), two recombinants (WAg526 and WAg529) had insertions in a different region of the chromosome (Fig. 3b) and the remaining three recombinants had insertions in widely separated parts of the chromosome (Fig. 3c-e). None of the insertions was in the same regions as those of the four recombinants produced in our earlier work (Wilson et al., 1997) and none of the deletions overlaps any of the blocks of genes that have been shown to be present in some strains of the M. tuberculosis complex, but absent in M. bovis or other strains of the complex (Behr et al., 1999; Gordon et al., 1999). The region in which the fragments had inserted did not appear to depend on the fragment used, as recombinants in the Rv0209-Rv0216 region (Fig. 3a) were produced by both fragments. As in our earlier work, the amount of gene 
Table 2. Details of the eight selected recombinants and the parent strain

\begin{tabular}{|c|c|c|c|c|c|}
\hline $\begin{array}{l}\text { Strain } \\
\text { (WAg) }\end{array}$ & $\begin{array}{c}\text { Fragment } \\
\text { used }\end{array}$ & $\begin{array}{l}\text { Mean no. of spleen } \\
\text { lesions per animal }\end{array}$ & $\begin{array}{l}\text { Deletion size } \\
\text { (bp) }\end{array}$ & $\begin{array}{l}\text { No. of genes } \\
\text { interrupted }\end{array}$ & Deletion region \\
\hline 525 & $R v 3844$ & 34 & 214 & 1 & 34004-34217 of Mty13E12 \\
\hline 526 & $R v 3844$ & 0 & 11227 & 12 & $16862-28088$ of MtcI28 \\
\hline 529 & $R v 3844$ & 2 & 7123 & 8 & 19999-27121 of MtcI28 \\
\hline 527 & $a h p C$ & 0 & 0 & 1 & Insert at 5424 in Mtcy8D5 \\
\hline 528 & $R v 3844$ & 0 & 10860 & 9 & 2270-13129 of Mtcy8D5 \\
\hline 531 & $a h p C$ & 0 & 2909 & 2 & 4354-7262 of Mtcy8D5 \\
\hline 530 & $a h p C$ & 0 & 30 & 1 & $5330-5359$ of Mtcy427 \\
\hline 532 & $a h p C$ & 38 & 3989 & 2 & 4628-8616 of Mtv035 \\
\hline Parent & - & 65 & - & - & - \\
\hline
\end{tabular}

(a)

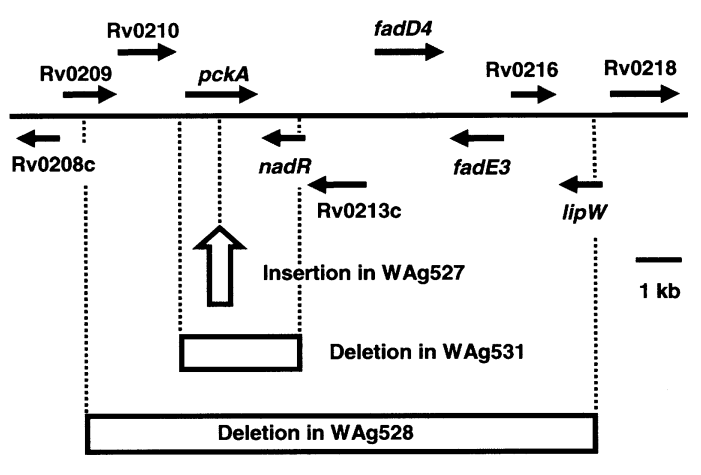

(b)

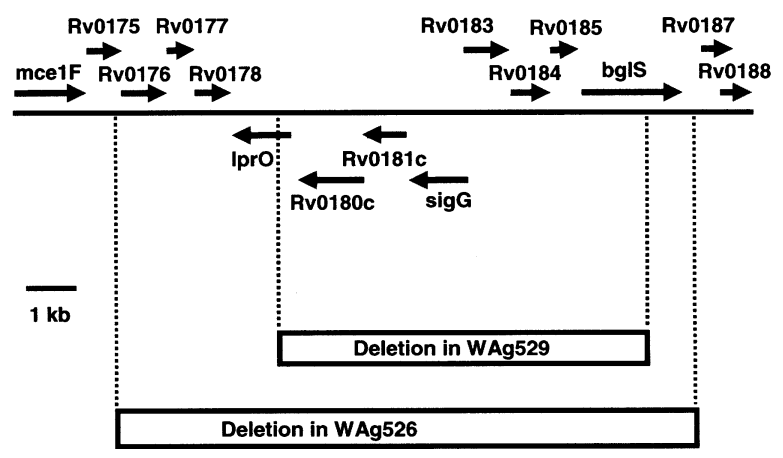

(c)

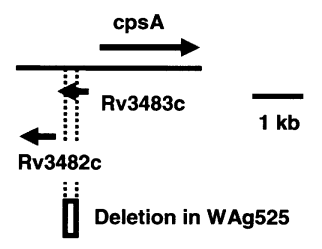

(d)

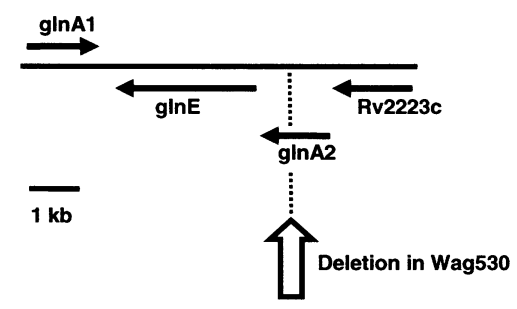

(e)

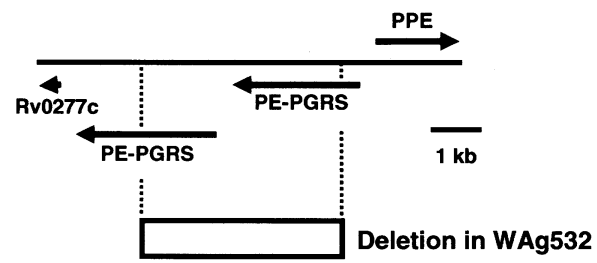

Fig. 3. Sites of illegitimate recombination in $M$. bovis of the eight selected recombinants. (a) WAg527, WAg528 and WAg531; (b) WAg526 and WAg529; (c) WAg525; (d) WAg530; (e) WAg532. Deletion and insertion are shown relative to the annotation of $M$. tuberculosis H37Rv.

deletion varied widely from $11 \mathrm{~kb}$ to no deletion (WAg527) and in some cases there had also been large deletions from the end of the inserting fragment. In the case of WAg527, parts of the NotI sites delineating the inserting fragment were still present and the insertion was accompanied by a chromosomal duplication of $9 \mathrm{bp}$ so that each end of the insertion was flanked by the same $9 \mathrm{bp}$ sequence. Although the inserting fragment has no homology to transposases, this duplication is very reminiscent of the $8 \mathrm{bp}$ duplications without deletion that occur on transposition of Tn5367 into the chromosome of the M. tuberculosis complex (Bardarov et al., 1997). Interestingly, the duplicated $9 \mathrm{bp}$ sequence in WAg527 contains a GGCGCC palindrome which has been shown to be a highly preferred recombination site for illegitimate recombination in Rhodococcus fascians (Desomer et al., 1991), although in that study no duplication at the sites of recombination occurred. None 
Table 3. Vaccination results

a,b,c,d, Values within the same column with the same suffix are not significantly different; ND, not determined.

\begin{tabular}{|lcccc|}
\hline $\begin{array}{l}\text { M. bovis } \\
\text { recombinant }\end{array}$ & $\begin{array}{c}\text { Mean spleen } \\
\text { weight } \pm \text { SE }(\mathrm{g})\end{array}$ & $\begin{array}{c}\text { Mean no. of spleen } \\
\text { lesions } \pm \text { SE }\end{array}$ & $\begin{array}{c}\log (\text { Spleen M. bovis } \\
\text { c.f.u. }) \pm \text { SE }\end{array}$ & $\begin{array}{c}\log (\text { Lung M. bovis } \\
\text { c.f.u. }) \pm S E\end{array}$ \\
\hline Non-vaccinated & $2 \cdot 50 \pm 0 \cdot 33^{\mathrm{a}}$ & $46 \pm 10^{\mathrm{a}}$ & $4 \cdot 97 \pm 0 \cdot 17^{\mathrm{a}}$ & $4 \cdot 22 \pm 0 \cdot 25^{\mathrm{a}}$ \\
BCG & $1 \cdot 29 \pm 0 \cdot 09^{\mathrm{c}}$ & $0 \cdot 25 \pm 0 \cdot 18^{\mathrm{d}}$ & $1 \cdot 53 \pm 0 \cdot 53^{\mathrm{c}}$ & $2 \cdot 36 \pm 0 \cdot 36^{\mathrm{b}}$ \\
WAg526 & $1 \cdot 17 \pm 0 \cdot 10^{\mathrm{c}}$ & $0 \cdot 0 \pm 0 \cdot 0^{\mathrm{d}}$ & $1 \cdot 21 \pm 0 \cdot 21^{\mathrm{c}}$ & $2 \cdot 41 \pm 0 \cdot 47^{\mathrm{b}}$ \\
WAg527 & $1 \cdot 82 \pm 0 \cdot 16^{\mathrm{bc}}$ & $26 \pm 17^{\mathrm{bc}}$ & $3 \cdot 46 \pm 0 \cdot 37^{\mathrm{b}}$ & $4 \cdot 19 \pm 0 \cdot 25^{\mathrm{a}}$ \\
WAg528 & $1 \cdot 97 \pm 0 \cdot 27^{\mathrm{ab}}$ & $36 \pm 12^{\mathrm{ab}}$ & $3 \cdot 40 \pm 0 \cdot 69^{\mathrm{b}}$ & $3 \cdot 83 \pm 0 \cdot 16^{\mathrm{a}}$ \\
WAg531 & $1 \cdot 35 \pm 0 \cdot 17^{\mathrm{bc}}$ & $1 \cdot 7 \pm 1 \cdot 3^{\mathrm{cd}}$ & $\mathrm{ND}$ & $\mathrm{ND}$ \\
WAg530 & $1 \cdot 15 \pm 0 \cdot 10^{\mathrm{c}}$ & $0 \cdot 0 \pm 0 \cdot 0^{\mathrm{d}}$ & $\mathrm{ND}$ & $\mathrm{ND}$ \\
\hline
\end{tabular}

of the other seven M. bovis recombinants had this palindrome at or within $9 \mathrm{bp}$ of their insertion sites.

\section{Analysis of mutations in relation to virulence}

The mean number of spleen lesions per guinea pig was used to indicate the virulence of the recombinants (Table 2 ). Five of the recombinants caused no spleen lesions and were used for vaccination studies. One of the recombinants had greatly reduced virulence and the remaining two recombinants had a level of virulence about half that of the parent strain (Table 2). Lesions in the liver were also recorded but were less sensitive for assessing virulence. None of the five recombinants in Table 3 produced any liver lesions, the parent strains produced a mean of three to five liver lesions per animal and WAg525 and WAg532 produced liver lesions in one guinea pig each. No delayed type hypersensitivity reactions were observed when the animals were skintested with PPD prior to being inoculated with the $M$. bovis recombinants. All animals had positive skin test reactions immediately prior to sacrifice, indicating that they had been successfully infected.

WAg527, WAg528 and WAg531 were all avirulent and all have an inactivated $p c k A$ gene. In the case of WAg527, this is the only gene inactivated and the gene annotation of this region (Fig. 3a) indicates that polar effects on nearby genes are unlikely. The avirulence of WAg528 and WAg531 could also be due entirely to the inactivation of $p c k A$, but the other known and unknown genes that are also inactivated in these two recombinants may also affect their virulence. $p c k A$ encodes phosphoenolpyruvate carboxykinase that catalyses the decarboxylation and phosphorylation of oxaloacetate to form phosphoenolpyruvate. This is the first committed step of gluconeogenesis in bacteria and the enzyme is utilized during gluconeogenic growth when sugar levels are low (Riedel et al., 2001; Matte et al., 1997). The inability of WAg527 to cause any disease indicates that the gluconeogenic pathway is essential for the virulence of $M$. bovis in guinea pigs.

WAg526 and WAg529 both have large deletions at the
sigG locus (Fig. 3b). WAg529 had greatly reduced virulence and WAg526 was avirulent. Since the deletion in WAg529 is nested within that of WAg526, it can be concluded that one or more of the extra genes of unknown function that were deleted in WAg526 ( $R v 0175-R v 0178)$ contribute to loss of virulence as well as at least one of the genes absent in both recombinants. Of the two genes of known function identified as inactivated in both recombinants, $\operatorname{sig} G$ is a sigma factor gene belonging to the family which regulates extracytoplasmic functions and stress responses and $b g l S$ encodes a $\beta$-glucosidase. Neither of these genes has been studied in detail but it has been reported that the expression of $\operatorname{sig} G$ is strongly down-regulated under conditions of low aeration (Manganelli et al., 1999). The 5- to 10-week-old cultures used for screening would have had lowered levels of oxygen and the inability of these two strains to grow when transferred to fresh minimal medium as well as their considerable loss of virulence may indicate that $\operatorname{sig} G$ plays a significant role when the bacteria are returning from lower to higher levels of oxygen, a situation that may also occur in vivo.

WAg530 has a small deletion in the $g \ln A 2$ gene encoding a putative glutamine synthetase and was found to be avirulent. Two other genes in the same chromosomal region (Fig. 3d), $g \ln A 2$ and $g \ln E$, are also involved in glutamine synthesis. The glutamine synthetase encoded by $g \ln A 1$, which has been extensively studied, is not actively exported from $M$. tuberculosis, but is found in the extracellular medium of M. tuberculosis cultures and may play a role in pathogenesis (Tullius et al., 2001). The product of $g \ln E$ is a regulator that by comparison to homologous enzymes in Streptomyces coelicolor controls $g \ln A 1$, but not $g \ln A 2$ (Parish \& Stoker, 2000). $g \ln E$ has been shown to be essential (Parish et al., 2001), but $g \ln A 2$ has not been studied. In the annotation of DNA sequences from $M$. tuberculosis, glnA2 reads in the same direction as $g \ln E$ and its coding sequence ends only $48 \mathrm{bp}$ before the initiation codon of $g \ln E$. This region is identical in the $M$. bovis genome. This raised the possibility that these two genes may be in an operon and may be expressed co-ordinately. To test this possibility, we measured RNA production at various points in this 
(a)

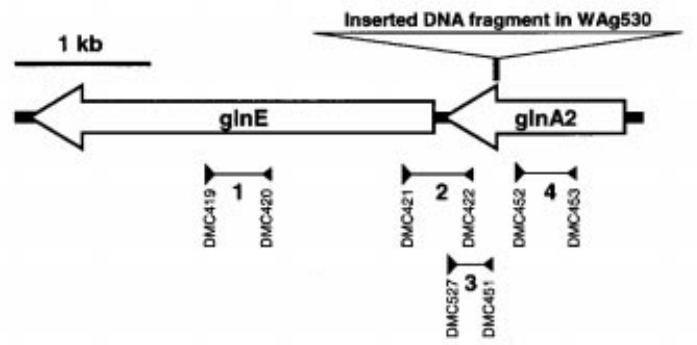

(b)

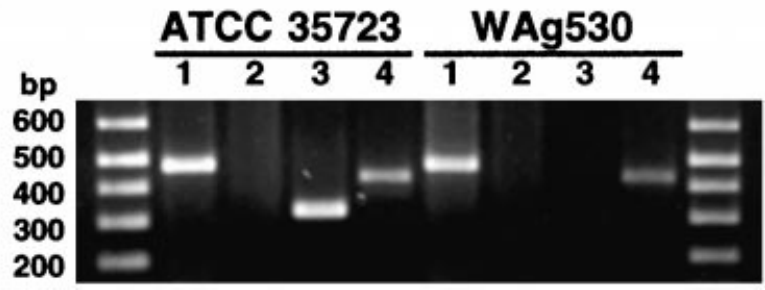

Fig. 4. Transcriptional characterization of $g \ln A 2$ and $g \ln E$ in $M$. bovis WAg530 and its parent strain ATCC 35723. (a) Alignment of the four primer pairs used for RT-PCR; large arrowheads indicate the primers used for reverse transcription. (b) Ethidium-stained agarose gel of PCR products from the four RTPCR reactions. Products of the expected size were obtained in all cases. There was no evidence of any product for primer pair 3 in Wag530. Control reactions (not shown) utilizing DNaseltreated RNA without reverse transcription failed to yield PCR products, while all primer pairs gave the expected PCR product when used to amplify genomic DNA.

region of the chromosome (Fig. 4). RNA transcription of $g \ln A 2$ upstream of the insertion site and of $g \ln E$ occurred in both WAg530 and its parent strain and there was no transcript running from $g \ln A 2$ to $g \ln E$ in either strain. The only transcription difference between the strains was the absence in WAg530 of any $g \ln A 2$ transcription downstream of the illegitimate insertion site in $g \ln A 2$. These results indicate that $g \ln A 2$ and $g \ln E$ are not in an operon and are consistent with the earlier finding that $g \ln E$ is an essential gene (Parish et al., 2001). The loss of virulence in WAg530 is thus likely to be entirely due to disruption of $g \ln A 2$ and not to downstream polar effects on $g \ln E$.

One of the virulent recombinants, WAg525 (Fig. 3c), had a deletion in a small gene of unknown function and was not further analysed. In the other recombinant that retained virulence, WAg532 (Fig. 3e), two PE-PGRS genes had been largely deleted (Fig. 3e). Cole et al. (1998) found that these genes are part of a big subfamily of 61 genes that together with the other 38 members of the PE family and the unrelated 68 members of the PPE family make up about $10 \%$ of the genome. Their proposal that these families may play a role in influencing the host's immune responses has received some experimental support (Delogu \& Brennan, 2001) and the putative proteins of the two genes inactivated in WAg523 are highly similar at their N-terminal ends to a PE-PGRS protein that binds fibronectin and is recognized immunologically by tuberculosis patients (Espitia et al.,
1999). There is also evidence that inactivation of some of these genes can affect infection of macrophages (Brennan et al., 2001) and this also occurs when similar PGRS genes are inactivated in the related pathogen Mycobacterium marinum (Ramakrishnan et al., 2000). The genes inactivated in WAg523 clearly had a phenotypic effect expressed under the screening regime but this had little apparent effect on virulence of WAg523 in guinea pigs. The guinea pig model gives a measure of acute disease and one possible explanation is that, given the way the strain was selected, the mutation has affected the ability of the strain to cause reactivation tuberculosis, a property that could only be tested in a reactivation animal model such as the Cornell model (Colston \& Cox, 1999). A superficial argument against this explanation is that both of these genes are absent from $M$. tuberculosis CDC1551, a recent clinical isolate (Betts et al., 2000). However, the complement of genes differs between strains of the $M$. tuberculosis complex and the differences appear greater between M. tuberculosis and M. bovis strains than between different $M$. tuberculosis strains (Behr et al., 1999; Brosch et al., 2002; Gordon et al., 1999). This situation, together with the similarity between PE-PGRS genes, indicates a likelihood that the absence of particular PE-PGRS genes in one strain may be compensated by the presence of other genes.

\section{Vaccination study}

No delayed type hypersensitivity reactions were observed when the animals were tested prior to vaccination. All animals had positive skin test reactions immediately prior to sacrifice and there were no statistical differences between the responses of the groups of challenged animals, including the non-vaccinated group. Macroscopic lesions consistent with tuberculosis were observed in the lungs of the guinea pigs challenged with virulent $M$. bovis and the protection afforded by the vaccines against spread of infection was judged by the size of the spleens, the number of spleen lesions and the $\log$ (transformed c.f.u. of M. bovis) in the spleen and lung (Table 3). Cultures were not available for WAg530 and WAg531. There were a few liver lesions in most of the unvaccinated control animals, and in some animals vaccinated with WAg528, but they were not sufficient to make these groups significantly different from the other vaccinated groups that had no liver lesions. The five recombinants that were tested gave widely varying levels of protection against the spread of infection. WAg527 and WAg528 provided relatively poor protection, although the spleen c.f.u. were significantly different from controls. WAg531 gave intermediate protection. The best protection was provided by BCG, WAg526 and WAg530 and these could not be distinguished statistically $(P<0.05)$. No spleen lesions were found for WAg526 and WAg530.

As in our earlier study (Wilson et al., 1997), several recombinants have produced results that, while not significantly different from those for BCG, do have some indices of protection that indicate a lower spread of 
infection than that achieved by BCG. In some recent vaccine models of tuberculosis in which animals were first exposed to environmental mycobacteria, BCG provided little or no protection against tuberculosis (Brandt et al., 2002; Buddle et al., 2002), although in the second of those studies chemical mutants of M. bovis gave significant protection. These differences in protection may result from differences in persistence between BCG and other avirulent strains or from stimulation of a qualitatively different immune response. Whether the strains developed in this study would give significantly better protection than BCG if one of these more recent vaccine models was used or if the vaccination protocol was altered in some other way to exemplify the differences between protective vaccines requires further experimentation. The two recombinants that gave poorer protection (WAg527 and WAg528) are both strains that lack an active $p c k A$ gene. WAg531, which also lacks an active $p c k A$ gene, provided protection that was not significantly different to that provided by BCG, although it did allow a higher mean spleen weight and lesion count than BCG, WAg526 and WAg530. These results show that while inactivating pckA does create avirulent strains of $M$. bovis, those strains do not appear to have the potential to provide a better vaccine than BCG. More generally, it also shows that avirulent strains of the $M$. tuberculosis complex are not all able to stimulate a good level of protection against tuberculosis even when their inoculation into guinea pigs results in normal skin test reactivity.

The results described here support the approach used in this and the previous study (Wilson et al., 1997; de Lisle et al., 1999) of screening stationary-phase cultures for their ability to grow in minimal medium and testing the virulence and vaccine potential of strains identified by this selection. Together with our earlier study, a total of 12 recombinants $(0.8 \%)$ have been isolated from 1440 tested of which nine $(0.6 \%)$ are avirulent. None of the genes inactivated in these nine avirulent strains has been reported in other studies as being important for virulence of strains of the $M$. tuberculosis complex. Several of the recombinants provide protection against the spread of tuberculosis that, while not significantly different to that provided by BCG, results in slightly better indices of protection than BCG. Whether the recombinants produced here would provide better protection against tuberculosis than BCG if they were tested under more stringent conditions remains to be determined, but the current results encourage belief that a better live vaccine than BCG may be attainable.

\section{ACKNOWLEDGEMENTS}

This study was supported by a grant from the New Zealand Foundation of Research Science and Technology.

\section{REFERENCES}

Bardarov, S., Kriakov, J., Carriere, C., Yu, S., Vaamonde, C., McAdam, R. A., Bloom, B. R., Hatfull, G. F. \& Jacobs, W. R., Jr (1997). Conditionally replicating mycobacteriophages: a system for transposon delivery to Mycobacterium tuberculosis. Proc Natl Acad Sci U S A 94, 10961-10966.

Behr, M. A., Wilson, M. A., Gill, W. P., Salamon, H., Schoolnik, G. K., Rane, S. \& Small, P. M. (1999). Comparative genomics of BCG vaccines by whole-genome DNA microarray. Science 284, 1520-1523.

Betts, J. C., Dodson, P., Quan, S., Lewis, A. P., Thomas, P. J., Duncan, K. \& McAdam, R. A. (2000). Comparison of the proteome of Mycobacterium tuberculosis strain $\mathrm{H} 37 \mathrm{Rv}$ with clinical isolate CDC (1551). Microbiology 146, 3205-3216.

Bloom, B. R. \& Fine, P. E. M. (1994). The BCG experience: implications for future vaccines against tuberculosis. In $T u$ berculosis: Pathogenesis, Protection and Control, pp. 531-557. Edited by B. R. Bloom. Washington, DC: American Society for Microbiology.

Brandt, L., Feino, C. J., Weinreich, O. A., Chilima, B., Hirsch, P., Appelberg, R. \& Andersen, P. (2002). Failure of the Mycobacterium bovis BCG vaccine: some species of environmental mycobacteria block multiplication of BCG and induction of protective immunity to tuberculosis. Infect Immun 70, 672-678.

Brennan, M. J., Delogu, G., Chen, Y., Bardarov, S., Kriakov, J., Alavi, M. \& Jacobs, W. R., Jr (2001). Evidence that mycobacterial PE_PGRS proteins are cell surface constituents that influence interactions with other cells. Infect Immun 69, 7326-7333.

Brewer, T. F. \& Colditz, G. A. (1995). Relationship between Bacille Calmette-Guerin (BCG) strains and the efficacy of BCG vaccine in the prevention of tuberculosis. Clin Infect Dis 20, 126-135.

Brosch, R., Gordon, S. V., Marmiesse, M. \& 12 other authors (2002). A new evolutionary scenario for the Mycobacterium tuberculosis complex. Proc Natl Acad Sci U S A 99, 3684-3689.

Buddle, B. M., Wards, B. J., Aldwell, F. E., Collins, D. M. \& de Lisle, G. W. (2002). Influence of sensitisation to environmental mycobacteria on subsequent vaccination against bovine tuberculosis. Vaccine 20, 1126-1133.

Cole, S. T., Brosch, R., Parkhill, J. \& 38 other authors (1998). Deciphering the biology of Mycobacterium tuberculosis from the complete genome sequence. Nature 393, 537-544.

Collins, D. M. (2000). New tuberculosis vaccines based on attenuated strains of the Mycobacterium tuberculosis complex. Immunol Cell Biol 78, 342-348.

Collins, D. M. (2001). Virulence factors of Mycobacterium bovis. Tuberculosis 81, 97-102.

Colston, M. J. \& Cox, R. A. (1999). Mycobacterial growth and dormancy. In Mycobacteria: Molecular Biology and Virulence, pp. 198-219. Edited by C. Ratledge \& J. Dale. London : Blackwell Science.

Darzins, E. (1958). The Bacteriology of Tuberculosis. Minneapolis: University of Minnesota Press.

de Lisle, G. W., Wilson, T., Collins, D. M. \& Buddle, B. M. (1999). Vaccination of guinea pigs with nutritionally impaired avirulent mutants of Mycobacterium bovis protects against tuberculosis. Infect Immun 67, 2624-2626.

Delogu, G. \& Brennan, M. J. (2001). Comparative immune response to PE and PE_PGRS antigens of Mycobacterium tuberculosis. Infect Immun 69, 5606-5611.

Desomer, J., Crespi, M. \& Van Montagu, M. (1991). Illegitimate integration of non-replicative vectors in the genome of Rhodococcus fascians upon electrotransformation as an insertional mutagenesis system. Mol Microbiol 5, 2115-2124.

Espitia, C., Laclette, J. P., Mondragon-Palomino, M., Amador, A., Campuzano, J., Martens, A., Singh, M., Cicero, R., Zhang, Y. \& Moreno, C. (1999). The PE-PGRS glycine-rich proteins of 
Mycobacterium tuberculosis: a new family of fibronectin-binding proteins? Microbiology 145, 3487-3495.

Fine, P. E. (1995). Bacille Calmette-Guerin vaccines: a rough guide. Clin Infect Dis 20, 11-14.

Gordon, S. V., Brosch, R., Billault, A., Garnier, T., Eiglmeier, K. \& Cole, S. T. (1999). Identification of variable regions in the genomes of tubercle bacilli using bacterial artificial chromosome arrays. Mol Microbiol 32, 643-655.

Hondalus, M. K., Bardarov, S., Russell, R., Chan, J., Jacobs, W. R., Jr \& Bloom, B. R. (2000). Attenuation of and protection induced by a leucine auxotroph of Mycobacterium tuberculosis. Infect Immun 68, 2888-2898.

Horwitz, M. A., Harth, G., Dillon, B. J. \& Maslesa-Galic, S. (2000). Recombinant Bacillus Calmette-Guerin (BCG) vaccines expressing the Mycobacterium tuberculosis $30-\mathrm{kDa}$ major secretory protein induce greater protective immunity against tuberculosis than conventional BCG vaccines in a highly susceptible animal model. Proc Natl Acad Sci U S A 97, 13853-13858.

Jackson, M., Phalen, S. W., Lagranderie, M., Ensergueix, D., Chavarot, P., Marchal, G., McMurray, D. N., Gicquel, B. \& Guilhot, C. (1999). Persistence and protective efficacy of a Mycobacterium tuberculosis auxotroph vaccine. Infect Immun 67, 2867-2873.

Mahairas, G. G., Sabo, P. J., Hickey, M. J., Singh, D. C. \& Stover, C. K. (1996). Molecular analysis of genetic differences between Mycobacterium bovis BCG and virulent M. bovis. J Bacteriol 178, 1274-1282.

Manganelli, R., Dubnau, E., Tyagi, S., Kramer, F. R. \& Smith, I. (1999). Differential expression of 10 sigma factor genes in Mycobacterium tuberculosis. Mol Microbiol 31, 715-724.

Matte, A., Tari, L. W., Goldie, H. \& Delbaere, L. T. J. (1997). Structure and mechanism of phosphoenolpyruvate carboxykinase. J Biol Chem 272, 8105-8108.

McMurray, D. N., Carlomagno, M. A., Mintzer, C. L. \& Tetzlaff, C. L. (1985). Mycobacterium bovis BCG vaccine fails to protect protein-deficient guinea pigs against respiratory challenge with virulent Mycobacterium tuberculosis. Infect Immun 50, 555-559.

Parish, T. \& Stoker, N. G. (2000). $g \ln E$ is an essential gene in Mycobacterium tuberculosis. J Bacteriol 182, 5715-5720.

Parish, T., Lewis, J. \& Stoker, N. G. (2001). Use of the mycobacteriophage L5 excisionase in Mycobacterium tuberculosis to demonstrate gene essentiality. Tuberculosis 81, 359-364.

Ramakrishnan, L., Federspiel, N. A. \& Falkow, S. (2000). Granuloma-specific expression of Mycobacterium virulence proteins from the glycine-rich PE-PGRS family. Science 288, 1436-1439.
Riedel, C., Rittmann, D., Dangel, P., Mockel, B., Petersen, S., Sahm, H. \& Eikmanns, B. J. (2001). Characterization of the phosphoenolpyruvate carboxykinase gene from Corynebacterium glutamicum and significance of the enzyme for growth and amino acid production. J Mol Microbiol Biotechnol 3, 573-583.

Snewin, V., Stewart, G. \& Young, D. (2000). Genetic strategies for vaccine development. In Molecular Genetics of Mycobacteria, pp. 279-296. Edited by G. F. Hatfull \& W. R. Jacobs, Jr. Washington, DC: American Society for Microbiology.

Tullius, M. V., Harth, G. \& Horwitz, M. A. (2001). High extracellular levels of Mycobacterium tuberculosis glutamine synthetase and superoxide dismutase in actively growing cultures are due to high expression and extracellular stability rather than to a protein-specific export mechanism. Infect Immun 69, 6348-6363.

van Soolingen, D., Hermans, P. W. M., de Haas, P. E. W., Soll, D. R. \& van Embden, J. D. A. (1991). Occurrence and stability of insertion sequences in Mycobacterium tuberculosis complex strains: evaluation of an insertion-sequence dependent DNA polymorphism as a tool in the epidemiology of tuberculosis. $J$ Clin Microbiol 29, 2578-2586.

Wards, B. J. \& Collins, D. M. (1996). Electroporation at elevated temperatures substantially improves transformation efficiency of slow-growing mycobacteria. FEMS Microbiol Lett 145, 101-105.

Wards, B. J., de Lisle, G. W. \& Collins, D. M. (2000). An esat6 knockout mutant of Mycobacterium bovis produced by homologous recombination will contribute to the development of a live tuberculosis vaccine. Tubercle Lung Dis 80, 185-189.

Wiegeshaus, E. H., McMurray, D. N., Grover, A. A., Harding, G. E. \& Smith, D. W. (1970). Host-parasite relationships in experimental airborne tuberculosis III. Relevance of microbial enumeration to acquired resistance in guinea pigs. Amer Rev Resp Dis 102, 422-429.

Wilson, T. M., de Lisle, G. W. \& Collins, D. M. (1995). Effect of inhA and katG on isoniazid-resistance and virulence of $\mathrm{Myco-}$ bacterium bovis. Mol Microbiol 15, 1009-1015.

Wilson, T., Wards, B. J., White, S. J., Skou, B., de Lisle, G. W. \& Collins, D. M. (1997). Production of avirulent Mycobacterium bovis strains by illegitimate recombination with DNA fragments containing an interrupted $a h p C$ gene. Tubercle Lung Dis 78, 229-235.

Received 26 March 2002; revised 5 July 2002; accepted 8 July 2002. 